\title{
Lorentz-covariant coordinate-space representation of the leading hadronic contribution to the anomalous magnetic moment of the muon
}

\author{
Harvey B. Meyer ${ }^{\mathrm{a}}$ \\ PRISMA Cluster of Excellence, Institut für Kernphysik and Helmholtz Institut Mainz, Johannes Gutenberg-Universität Mainz, \\ 55099 Mainz, Germany
}

Received: 14 June 2017 / Accepted: 4 September 2017 / Published online: 16 September 2017

(C) The Author(s) 2017. This article is an open access publication

\begin{abstract}
We present a Lorentz-covariant, Euclidean coordinate-space expression for the hadronic vacuum polarisation, the Adler function and the leading hadronic contribution to the anomalous magnetic moment of the muon. The representation offers a high degree of flexibility for an implementation in lattice QCD. We expect it to be particularly helpful for the quark-line disconnected contributions.
\end{abstract}

\section{Introduction}

Two-point functions of the quark-flavor currents $\bar{q} \gamma_{\mu} q$ play an importance role in precision tests of the Standard Model of particle physics. The electromagnetic current $j_{\mu}^{\mathrm{em}}$ correlator enters the running of the QED coupling constant, and the non-diagonal correlation of $j_{\mu}^{\mathrm{em}}$ with the weak-isospin current $j_{\mu}^{3}$ contributes to the running of the weak mixing angle [1]. Furthermore, the leading hadronic contribution to the anomalous magnetic moment of the muon $a_{\mu}^{\text {hvp }}$ can be determined by non-perturbative theory methods from the electromagnetic current correlator. The contribution $a_{\mu}^{\text {hvp }}$, where 'HVP' stands for hadronic vacuum polarization, represents the largest uncertainty in the Standard Model prediction for this precision observable. Given the experiments in preparation [2] at FermiLab [3] and JPARC [4], which are expected to improve the accuracy of the direct measurement by a factor 4 , it is important to reduce the uncertainty on the prediction by a comparable factor. While the phenomenolog-

\footnotetext{
a e-mail: meyerh@uni-mainz.de
}

ical determination of $a_{\mu}^{\text {hvp }}$ via its dispersive representation is still the most accurate approach $[5,6]$, a purely theoretical prediction is both conceptually desirable and provides for an independent check. Since the vacuum polarization is inserted into an integral which is strongly weighted to the low-energy domain, calculating the hadronic vacuum polarization has become an important goal for several lattice QCD collaborations [7-18].

In lattice QCD, which representation of the $a_{\mu}^{\text {hvp }}$ is used matters, since the choice affects the systematic and statistical uncertainties of the result. In the pioneering article [7], $a_{\mu}^{\text {hvp }}$ was written as an integral of the vacuum polarization $\Pi\left(Q^{2}\right)$ over all virtualities $Q^{2}$. Later, other representations were proposed, in particular the time-moment representation [19-21], where the vector correlation function is projected onto vanishing spatial momentum and integrated as a function of Euclidean time. In this article we present a manifestly Lorentz-invariant representation of the vacuum polarization $\Pi\left(Q^{2}\right)$, the Adler function $Q^{2} \Pi^{\prime}\left(Q^{2}\right)$ and $a_{\mu}^{\text {hvp }}$ based on the coordinate-space representation of the vector correlator. We call it the covariant coordinate-space (CCS) representation. Besides its formal elegance, we expect it to be helpful in lattice QCD calculations, as the Lorentz symmetry present in the continuum leads to a high degree of flexibility and opportunities for cross-checks in its implementation. We think it will be especially beneficial for disconnecteddiagram contributions [14,22-24], where, in the standard algorithm, the noise-to-signal ratio on the coordinate-space correlator increases rapidly at long distances.

The structure of this paper is as follows. Our basic definitions and the derivation of the position-space expressions are presented in the next section. A test and illustration of the method is also provided. Section 3 is devoted to some aspects of the implementation of the method in lattice QCD. 


\section{Derivation of the covariant coordinate-space expressions}

In this section we consider continuum QCD in infinite Euclidean space. The conserved vector current is defined as $j_{\mu}(x)=\bar{\psi}(x) \gamma_{\mu} \psi(x)$, where the Dirac matrices are all hermitian and satisfy $\left\{\gamma_{\mu}, \gamma_{\nu}\right\}=2 \delta_{\mu \nu}$.

\subsection{Definitions}

The primary object is the position-space correlator

$G_{\mu \nu}(x)=\left\langle j_{\mu}(x) j_{\nu}(0)\right\rangle$.

The polarization tensor is its Fourier transform,

$\Pi_{\mu \nu}(Q) \equiv \int d^{4} x e^{i Q \cdot x} G_{\mu \nu}(x)$,

and $O(4)$ invariance and current conservation imply the tensor structure

$\Pi_{\mu \nu}(Q)=\left(Q_{\mu} Q_{\nu}-\delta_{\mu \nu} Q^{2}\right) \Pi\left(Q^{2}\right)$.

With these conventions, the spectral function

$\rho\left(q^{2}\right) \equiv-\left.\frac{1}{\pi} \operatorname{Im} \Pi\left(Q^{2}\right)\right|_{Q_{0}=-i q_{0}+\epsilon, \boldsymbol{Q}=\boldsymbol{q}}$

is non-negative for a flavor-diagonal correlator. For the electromagnetic current, it is related to the $R$ ratio via

$\rho(s)=\frac{R(s)}{12 \pi^{2}}, \quad R(s) \equiv \frac{\sigma\left(e^{+} e^{-} \rightarrow \text { hadrons }\right)}{4 \pi \alpha(s)^{2} /(3 s)}$.

The denominator is the treelevel cross-section $\sigma\left(e^{+} e^{-} \rightarrow\right.$ $\mu^{+} \mu^{-}$) in the limit $s \gg m_{\mu}^{2}$, and we have neglected QED corrections. The vacuum polarization, and the Adler function

$\mathcal{A}\left(Q^{2}\right) \equiv Q^{2} \frac{\mathrm{d}}{\mathrm{d} Q^{2}} \Pi\left(Q^{2}\right)$,

are recovered through a dispersion relation,

$$
\begin{aligned}
\Pi\left(Q^{2}\right)-\Pi(0) & =Q^{2} \int_{0}^{\infty} \mathrm{d} s \frac{\rho(s)}{s\left(s+Q^{2}\right)}, \\
\mathcal{A}\left(Q^{2}\right) & =Q^{2} \int_{0}^{\infty} \mathrm{d} s \frac{\rho(s)}{\left(s+Q^{2}\right)^{2}} .
\end{aligned}
$$

\subsection{Derivation}

As a motivation, we start from the expression [25] for $a_{\mu}^{\text {hvp }}$ in terms of the Adler function,

$a_{\mu}^{\mathrm{hvp}}=2 \pi^{2}\left(\frac{\alpha}{\pi}\right)^{2} \int_{0}^{1} \frac{\mathrm{d} y}{y}(1-y)(2-y) \mathcal{A}\left(Q^{2}(y)\right)$,

where

$$
Q^{2}(y)=\frac{y^{2}}{1-y} m_{\mu}^{2} \leftrightarrow \quad y=\frac{2|Q|}{|Q|+\sqrt{4 m_{\mu}^{2}+Q^{2}}} .
$$

Returning to the integration variable $Q^{2}$, we obtain

$$
\begin{aligned}
& a_{\mu}^{\mathrm{hvp}}=\int_{0}^{\infty} \mathrm{d} Q^{2} \mathcal{A}\left(Q^{2}\right) g_{a}\left(Q^{2}\right), \\
& g_{a}\left(Q^{2}\right)=2 \alpha^{2} \frac{m_{\mu}^{4}}{|Q|^{6}} y(|Q|)^{4} .
\end{aligned}
$$

However, we will keep the derivation more general and consider a general Lorentz-scalar physical quantity derived from the vector correlator,

$\Phi[g]=\int_{0}^{\infty} \mathrm{d} Q^{2} \mathcal{A}\left(Q^{2}\right) g\left(Q^{2}\right)$,

for some function $g\left(Q^{2}\right)$. Below, we reinterpret Eq. (13) as a four-dimensional integral with spherical symmetry, ${ }^{1}$

$\Phi[g]=\frac{1}{\pi^{2}} \int \frac{\mathrm{d}^{4} Q}{Q^{2}} \mathcal{A}\left(Q^{2}\right) g\left(Q^{2}\right)$.

We project out the transverse component of the polarisation tensor,

$\pi_{T}(Q)=\left(\delta_{\mu \nu}-\frac{Q_{\mu} Q_{\nu}}{Q^{2}}\right) \Pi_{\mu \nu}(Q)=-3 Q^{2} \Pi\left(Q^{2}\right)$.

The Adler function can be expressed via $\pi_{T}(Q)$ via

$\mathcal{A}\left(Q^{2}\right)=\frac{1}{3 Q^{2}}\left[\pi_{T}(Q)-\frac{Q_{\lambda}}{2} \frac{\partial}{\partial Q_{\lambda}} \pi_{T}(Q)\right]$

Inserting the position-space correlator,

$\pi_{T}(Q)=\left(\delta_{\mu \nu}-\frac{Q_{\mu} Q_{\nu}}{Q^{2}}\right) \int \mathrm{d}^{4} x G_{\mu \nu}(x) e^{i Q \cdot x}$,

we obtain

$$
\begin{aligned}
\mathcal{A}\left(Q^{2}\right)= & \frac{1}{3 Q^{2}}\left(\delta_{\mu \nu}-\frac{Q_{\mu} Q_{\nu}}{Q^{2}}\right) \\
& \times \int \mathrm{d}^{4} x G_{\mu \nu}(x) \mathrm{e}^{i Q \cdot x}\left(1-\frac{i}{2}(Q \cdot x)\right) .
\end{aligned}
$$

Inserting this expression into Eq. (14) and interchanging the order of the momentum-space and position-space integrals, one reaches

$$
\Phi[g]=\int \mathrm{d}^{4} x G_{\mu \nu}(x) H_{\mu \nu}(x),
$$

$H_{\mu \nu}(x)=\frac{1}{3 \pi^{2}}\left(1-\frac{x_{\lambda}}{2} \frac{\partial}{\partial x_{\lambda}}\right) I_{\mu \nu}(x)$,

$$
I_{\mu \nu}(x)=\int \frac{\mathrm{d}^{4} Q}{\left(Q^{2}\right)^{2}} g\left(Q^{2}\right)\left(\delta_{\mu \nu}-\frac{Q_{\mu} Q_{\nu}}{Q^{2}}\right) \mathrm{e}^{i Q \cdot x} .
$$

The kernel $I_{\mu \nu}(x)$ can be expressed as

$$
I_{\mu \nu}(x)=\left(\partial_{\mu}^{(x)} \partial_{\nu}^{(x)}-\delta_{\mu \nu} \triangle_{x}\right) I\left(x^{2}\right),
$$

\footnotetext{
${ }^{1}$ The unit sphere in four dimensions has a surface of $2 \pi^{2}$.
} 


$$
\begin{aligned}
I\left(x^{2}\right) & =\int \frac{\mathrm{d}^{4} Q}{\left(Q^{2}\right)^{3}} g\left(Q^{2}\right) \mathrm{e}^{i Q \cdot x} \\
& =\frac{4 \pi^{2}}{|x|} \int_{0}^{\infty} \frac{\mathrm{d}|Q|}{|Q|^{4}} g\left(Q^{2}\right) J_{1}(|Q||x|),
\end{aligned}
$$

where we performed the angular integration of the Fourier transform and the $J_{n}(z)$ are the Bessel functions of the first kind. Using the chain rule, we obtain

$$
\begin{aligned}
I_{\mu \nu}(x)= & -\delta_{\mu \nu}\left(\frac{\partial^{2} I}{\partial|x|^{2}}+\frac{2}{|x|} \frac{\partial I}{\partial|x|}\right) \\
& +\frac{x_{\mu} x_{\nu}}{x^{2}}\left(\frac{\partial^{2} I}{\partial|x|^{2}}-\frac{1}{|x|} \frac{\partial I}{\partial|x|}\right) .
\end{aligned}
$$

and

$$
\begin{gathered}
\left(1-\frac{x_{\lambda}}{2} \frac{\partial}{\partial x_{\lambda}}\right) I_{\mu \nu}(x)=-\delta_{\mu \nu}\left(-\frac{|x|}{2} \frac{\partial^{3} I}{\partial|x|^{3}}+\frac{3}{|x|} \frac{\partial I}{\partial|x|}\right) \\
+\frac{x_{\mu} x_{\nu}}{x^{2}}\left(-\frac{|x|}{2} \frac{\partial^{3} I}{\partial|x|^{3}}+\frac{3}{2} \frac{\partial^{2} I}{\partial|x|^{2}}-\frac{3}{2|x|} \frac{\partial I}{\partial|x|}\right)
\end{gathered}
$$

We note that an $x$-independent term in $H_{\mu \nu}(x)$ would not contribute to $a_{\mu}^{\mathrm{hvp}}$, since

$$
\int \mathrm{d}^{4} x G_{\mu \nu}(x)=0 \quad \forall \mu, v
$$

in the vacuum, when all correlation lengths are finite. We make use of this property to subtract from $H_{\mu \nu}(x)$ an $x$ independent term proportional to $\delta_{\mu \nu}$. The derivatives with respect to $|x|$ act on $J_{1}(|Q||x|) /|x|$, resulting in Bessel functions of higher order. We define

$$
\begin{aligned}
h_{1}(z) & =\frac{3}{8}+\left(-\frac{z}{2} \frac{\partial^{3}}{\partial z^{3}}+\frac{3}{z} \frac{\partial}{\partial z}\right) \frac{J_{1}(z)}{z} \\
& =\frac{3}{8}+\frac{1}{2} J_{0}(z)-\frac{5}{2} \frac{J_{1}(z)}{z}+3 \frac{J_{2}(z)}{z^{2}} \\
h_{2}(z) & =\left(-\frac{z}{2} \frac{\partial^{3}}{\partial z^{3}}+\frac{3}{2} \frac{\partial^{2}}{\partial z^{2}}-\frac{3}{2 z} \frac{\partial}{\partial z}\right) \frac{J_{1}(z)}{z} \\
& =\frac{1}{2 z^{3}}\left(z\left(z^{2}-24\right) J_{0}(z)-8\left(z^{2}-6\right) J_{1}(z)\right) .
\end{aligned}
$$

We note that for $z \rightarrow 0, h_{i}(z) \sim \lambda_{i} z^{4}$ with

$$
\lambda_{1}=\frac{7}{3072}, \quad \lambda_{2}=\frac{1}{768} \text {. }
$$

Thus, inserting the explicit form of $g\left(Q^{2}\right)$, we obtain finally the physical quantity of interest $\Phi[g]=\int \mathrm{d}^{4} x G_{\mu \nu}(x) H_{\mu \nu}(x)$ with

$$
\begin{aligned}
& H_{\mu \nu}(x)=-\delta_{\mu \nu} \mathcal{H}_{1}(|x|)+\frac{x_{\mu} x_{v}}{x^{2}} \mathcal{H}_{2}(|x|), \\
& \mathcal{H}_{i}(|x|)=\frac{2}{3} \int_{0}^{\infty} \frac{\mathrm{d} Q^{2}}{Q^{2}} h_{i}(|Q||x|) g\left(Q^{2}\right) .
\end{aligned}
$$

Thus, for Eq. (19) to provide an explicit expression for $\Phi[g]$, it suffices to pre-compute the weight functions $\mathcal{H}_{i}(|x|)$. For now we note that once the spacetime indices of
$G_{\mu \nu}(x)$ and $H_{\mu \nu}(x)$ are contracted, the integral $\int d^{4} x \rightarrow$ $2 \pi^{2} \int_{0}^{\infty} \mathrm{d}|x||x|^{3}$ becomes one-dimensional. Secondly, since $I_{\mu \nu}(x)$ and $H_{\mu \nu}(x)$ are transverse tensors, $\partial_{\mu}^{(x)} H_{\mu \nu}(x)=0$, a relation exists between the weight functions,

$\mathcal{H}_{1}^{\prime}(|x|)=\mathcal{H}_{2}^{\prime}(|x|)+\frac{3 \mathcal{H}_{2}(|x|)}{|x|}$.

\subsubsection{Computing the Adler function}

To obtain the Adler function, $\Phi[g] \doteq \mathcal{A}\left(Q^{2}\right)$, we simply set

$g\left(Q_{\mathrm{ref}}^{2}\right) \doteq \delta\left(Q^{2}-Q_{\mathrm{ref}}^{2}\right)$,

and obtain immediately

$\mathcal{H}_{i}(|x|)=\frac{2 h_{i}(|Q||x|)}{3 Q^{2}}$.

A particularly elegant expression results in the limit $Q^{2} \rightarrow 0$ for the slope of the Adler function, or equivalently of the vacuum polarisation,

$$
\begin{aligned}
& \mathcal{A}^{\prime}(0)=\Pi^{\prime}(0) \\
& \quad=\frac{1}{1152} \int \mathrm{d}^{4} x G_{\mu \nu}(x)\left(x^{2}\right)^{2}\left(-\frac{7}{4} \delta_{\mu \nu}+\frac{x_{\mu} x_{\nu}}{x^{2}}\right) .
\end{aligned}
$$

Since it is well-known that the leading hadronic contribution to the anomalous magnetic moment of a lepton in the massless-lepton limit is given by the slope of the Adler function at the origin,

$\lim _{m_{\mu} \rightarrow 0} \frac{a_{\mu}^{\text {hvp }}}{m_{\mu}^{2}}=\frac{4}{3} \alpha^{2} \mathcal{A}^{\prime}(0)$,

Equation (37) can be used to calculate the hadronic contribution to the $(g-2)$ of the electron.

\subsubsection{The subtracted vacuum polarisation}

To obtain the subtracted vacuum polarisation,

$\Phi[g] \doteq \Pi\left(Q^{2}\right)-\Pi(0)$,

we set

$g\left(Q_{\mathrm{ref}}^{2}\right) \doteq \frac{1}{Q_{\mathrm{ref}}^{2}} \theta\left(Q^{2}-Q_{\mathrm{ref}}^{2}\right)$,

and obtain $\mathcal{H}_{i}(|x|)=x^{2} \cdot \overline{\mathcal{H}}_{i}(|Q||x|)$, where

$$
\begin{aligned}
\overline{\mathcal{H}}_{1}(z)= & \frac{z^{2}}{4608}\left\{24_{2} F_{3}\left(1,1 ; 2,3,3 ;-\frac{z^{2}}{4}\right)\right. \\
& -20_{2} F_{3}\left(1,1 ; 2,3,4 ;-\frac{z^{2}}{4}\right) \\
& \left.+3{ }_{2} F_{3}\left(1,1 ; 2,3,5 ;-\frac{z^{2}}{4}\right)\right\}
\end{aligned}
$$


and

$$
\begin{aligned}
\overline{\mathcal{H}}_{2}(z)= & \frac{z^{2}}{1152}\left\{6_{2} F_{3}\left(1,1 ; 2,3,3 ;-\frac{z^{2}}{4}\right)\right. \\
& -8{ }_{2} F_{3}\left(1,1 ; 2,3,4 ;-\frac{z^{2}}{4}\right) \\
& +4{ }_{2} F_{3}\left(1,1 ; 2,4,4 ;-\frac{z^{2}}{4}\right) \\
& \left.-{ }_{2} F_{3}\left(1,1 ; 2,4,5 ;-\frac{z^{2}}{4}\right)\right\} .
\end{aligned}
$$

The functions in the brackets are the generalized hypergeometric functions.

\subsubsection{The case of $a_{\mu}^{\text {hvp }}$}

Here we set $g\left(Q^{2}\right)=g_{a}\left(Q^{2}\right)$, and the weight functions can be written

$$
\begin{aligned}
\mathcal{H}_{i}(|x|) & =\frac{8 \alpha^{2}}{3 m_{\mu}^{2}} f_{i}\left(m_{\mu}|x|\right), \\
f_{i}\left(m_{\mu}|x|\right) & =m_{\mu}^{6} \int_{0}^{\infty} \frac{\mathrm{d}|Q|}{|Q|^{3}}\left(\frac{y(|Q|)}{|Q|}\right)^{4} h_{i}(|Q||x|) .
\end{aligned}
$$

To study the dimensionless weight functions $f_{i}(z)$, we perform the change of variables $\bar{Q}=|Q| / m_{\mu}$,

$f_{i}(z)=\int_{0}^{\infty} \frac{\mathrm{d} \bar{Q}}{\bar{Q}^{3}} \frac{16 h_{i}(z \bar{Q})}{\left(\bar{Q}+\sqrt{4+\bar{Q}^{2}}\right)^{4}}$.

Note that since the weight functions only depend on $m_{\mu}|x|$, the small $|x|$ behavior is at the same time the small $m_{\mu}$ behavior. Making use of the integral $\int_{0}^{\infty} \mathrm{d} v \frac{16 v}{\left(v+\sqrt{4+v^{2}}\right)^{4}}=\frac{1}{3}$, we obtain

$f_{i}(z) \stackrel{z \rightarrow 0}{=} \frac{\lambda_{i}}{3} z^{4}$.

Thus in the limit of vanishing lepton mass, we recover Eqs. (38-37).

The regime of large $|x|$ is more easily investigated from Eq. (44) by performing the change of variables $v=|Q||x|$,

$f_{i}(z)=z^{6} \int_{0}^{\infty} \frac{\mathrm{d} v}{v^{3}} \frac{16 h_{i}(v)}{\left(v+\sqrt{4 z^{2}+v^{2}}\right)^{4}}$.

Taking the limit of large argument (corresponding to large $|x|), f_{i}(z) \stackrel{z \rightarrow \infty}{=} v_{i} z^{2}$, with

$v_{i}=\int_{0}^{\infty} \frac{\mathrm{d} v}{v^{3}} h_{i}(v), \quad v_{1}=\frac{5}{192}, \quad v_{2}=\frac{1}{96}$.

By expanding the denominator of $f_{2}(z)$ further, one obtains the first terms of a series in $1 / z$,

$f_{2}(z)=\frac{z^{2}}{96}-\frac{z}{15}+\frac{1}{4}-\frac{5}{8 z}+\cdots$
The subleading terms cannot directly be obtained from the representation (47).

We now derive an expression for the weight function in terms of known special functions. From Eq. (34), since both weight functions are proportional to $z^{4}$ at small $z$, the integration yields

$f_{1}(z)=f_{2}(z)+3 \int_{0}^{z} \mathrm{~d} \bar{z} \frac{f_{2}(\bar{z})}{\bar{z}}$.

We will therefore first address $f_{2}(z)$, and then obtain $f_{1}(z)$ from this equation.

Suppose that we want to calculate a function $\phi(z)$, and that we are initially able to calculate the expression

$$
-L_{r} \phi(r) \equiv-\left(\frac{\mathrm{d}^{2}}{\mathrm{~d} r^{2}}+\frac{3}{r} \frac{\mathrm{d}}{\mathrm{d} r}\right) \phi(r)=\rho(r) .
$$

The differential operator $L_{r}$ appearing in this equation is nothing but the four-dimensional Laplacian in spherical coordinates, $r$ playing the role of the radial coordinate. We are then dealing with an electrostatic problem in $4+1$ dimensions for a spherically symmetric charge distribution. Therefore, assuming $\rho(r)$ falls off faster than $1 / r^{2}$ at large $r$, the function $\phi(r)$ can be obtained using the Green's function $G_{0}(x)=\frac{1}{4 \pi^{2} x^{2}}$,

$\phi(|x|)=\int \mathrm{d}^{4} y G_{0}(x-y) \rho(|y|)$.

Defining

$d_{0}(|x|,|y|)=\theta\left(x^{2}-y^{2}\right) \frac{1}{|x|^{2}}+\theta\left(y^{2}-x^{2}\right) \frac{1}{|y|^{2}}$,

the convolution integral can be simplified using Gauss's theorem and exploiting the spherical symmetry of $\rho(r)$,

$$
\begin{aligned}
\phi(|x|) & =\frac{1}{2} \int_{0}^{\infty} \mathrm{d}|y||y|^{3} \mathrm{~d}_{0}(|x|,|y|) \rho(|y|) \\
& =\frac{1}{2|x|^{2}} \int_{0}^{|x|} \mathrm{d}|y||y|^{3} \rho(|y|)+\frac{1}{2} \int_{|x|}^{\infty} \mathrm{d} y|y| \rho(|y|) .
\end{aligned}
$$

We start from Eq. (45), note that

$L_{z}\left(z^{2} h_{2}(z)\right)=\frac{z^{2}}{2} J_{2}(z)$,

and compute the integral

$$
\begin{aligned}
& L_{z}\left(z^{2} f_{2}(z)\right)=\frac{z^{2}}{2} \int_{0}^{\infty} \frac{\mathrm{d} \bar{Q}}{\bar{Q}} \frac{16 J_{2}\left(\bar{Q}_{z}\right)}{\left(\bar{Q}+\sqrt{4+\bar{Q}^{2}}\right)^{4}} \\
& =\frac{z^{2}}{2 \sqrt{\pi}} G_{2,4}^{2,2}\left(z^{2}{ }_{1,2,-2,-1}{ }^{\frac{1}{2}, 1}\right),
\end{aligned}
$$

where $G_{p, q}^{m, n}$ represents Meijer's function. In order to recover $f_{2}(z)$ itself, we would like to apply Eq. (54). However, 


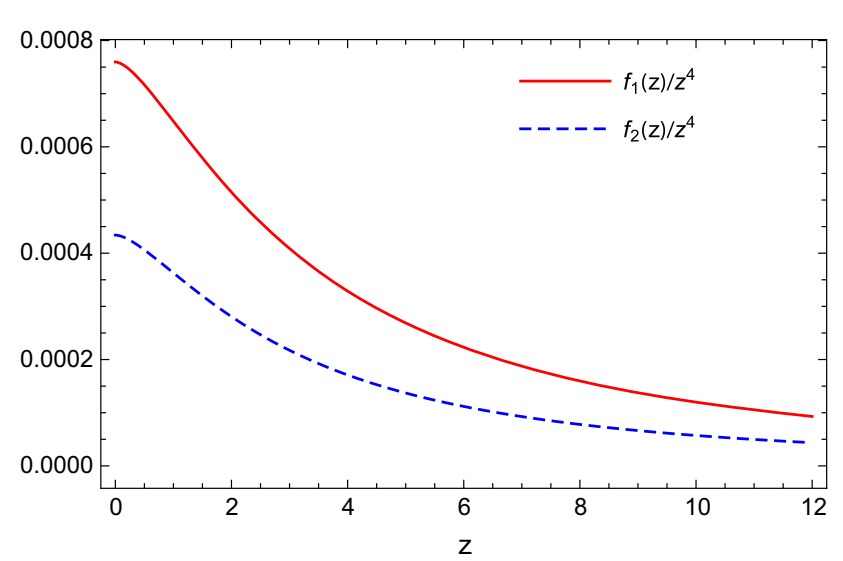

Fig. 1 The weight functions $f_{1}(z)$ and $f_{2}(z)$ needed to calculate $a_{\mu}^{\text {hvp }}$ using Eqs. $(19,33,43,62,61)$. In the calculation of $a_{\mu}^{\text {hvp }}$, the argument of the weight functions is $\left(m_{\mu}|x|\right)$, so that $z=1$ corresponds to $1.87 \mathrm{fm}$. Note that $\lim _{z \rightarrow 0} f_{1}(z) / z^{4}=\frac{7}{9216}$ and $\lim _{z \rightarrow 0} f_{1}(z) / z^{4}=\frac{1}{2304}$; see Eqs. (32) and (46)

$z^{2} f_{2}(z) \sim z^{4}$ at large $z$. We therefore perform subtractions using (49),

$f_{2}^{\mathrm{sub}}(z) \equiv f_{2}(z)-\left(\frac{z^{2}}{96}-\frac{z}{15}+\frac{1}{4}-\frac{5}{8 z}\right)$,

which obeys

$$
-L_{z}\left(z^{2} f_{2}^{\mathrm{sub}}(z)\right)=-L_{z}\left(z^{2} f_{2}(z)\right)+\frac{z^{2}}{4}-z+2-\frac{15}{8 z} .
$$

We thus obtain the representation

$$
z^{2} f_{2}^{\text {sub }}(z)=\frac{1}{2} \int_{0}^{\infty} \mathrm{d} \bar{z} \bar{z}^{3} \mathrm{~d}_{0}(z, \bar{z})\left(-L_{\bar{z}}\left(\bar{z}^{2} f_{2}^{\mathrm{sub}}(\bar{z})\right)\right) .
$$

After expression (56) is inserted into Eq. (59) and the latter in turn into Eq. (60), $f_{2}^{\text {sub }}(z)$ can be expressed again in terms of Meijer's function. Reintroducing the subtracted terms from (58), we finally obtain

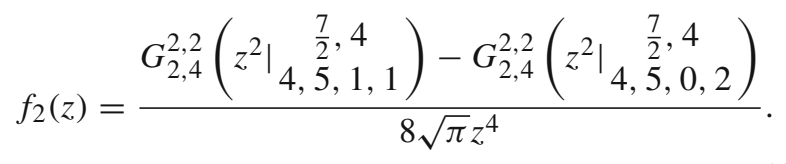

From Eq. (50), we then obtain

$$
\begin{aligned}
f_{1}(z)= & f_{2}(z)-\frac{3}{16 \sqrt{\pi}} \cdot\left[G _ { 3 , 5 } ^ { 2 , 3 } \left(\left.z^{2}\right|_{2,3,-2,0,0} 1, \frac{3}{2}, 2\right.\right. \\
& \left.-G_{3,5}^{2,3}\left(\left.z^{2}\right|_{2,3,-1,-1,0}\right)\right]
\end{aligned}
$$

The functions $f_{i}(z) / z^{4}$ are displayed in Fig. 1. The latter ratios depend very weakly on $\mathrm{z}$ in the range where they will be needed to compute $a_{\mu}^{\text {hvp }}$.

\subsection{Test of the CCS method}

To test the method, we construct a simple model for the position-space correlator $G_{\mu \nu}(x)$ based on its spectral representation. The latter reads

$$
\begin{aligned}
G_{\mu \nu}(x) & =\left(\partial_{\mu}^{(x)} \partial_{\nu}^{(x)}-\delta_{\mu \nu} \triangle_{x}\right) \tilde{\Pi}(|x|), \\
\tilde{\Pi}(|x|) & =\int_{0}^{\infty} \mathrm{d} s \rho(s) G_{\sqrt{s}}(x),
\end{aligned}
$$

where

$$
G_{m}(x)=\frac{m}{4 \pi^{2}|x|} K_{1}(m|x|)
$$

is the scalar propagator. The derivatives in Eq. (63) act like in Eq. (22), see Eq. (25). For illustration and testing purposes, we choose as a model

$\rho(s)=\frac{2}{3} f_{V}^{2} M^{2} \delta\left(s-M^{2}\right)$.

We note that the physical quantities to be computed are linear functions of the spectral function $\rho(s)$, and in a finite-volume system the most general spectral function is a linear combination of contributions of the type (66). We remark that in the latter equation, the decay constant $f_{V}$ is dimensionless. Then, with $r=|x|$, we obtain

$$
\begin{aligned}
& G_{\mu \nu}(x) \\
& =\frac{f_{V}^{2} M^{3}}{6 \pi^{2}}\left[-\delta_{\mu \nu} \frac{M}{r^{2}}\left(K_{2}(M r)+M r K_{1}(M r)\right)\right. \\
& \left.\quad+\frac{x_{\mu} x_{v}}{x^{2}} \frac{1}{r^{3}}\left(4 M r K_{0}(M r)+\left(M^{2} r^{2}+8\right) K_{1}(M r)\right)\right] .
\end{aligned}
$$

In general, if the position-space correlator is written in the form

$G_{\mu \nu}(x)=-\delta_{\mu \nu} \mathcal{G}_{1}(|x|)+\frac{x_{\mu} x_{v}}{x^{2}} \mathcal{G}_{2}(|x|)$,

physical quantities derived from it can be obtained from the scalar integral

$\Phi[g]=2 \pi^{2} \int_{0}^{\infty} \mathrm{d} r r^{3}\left[\mathcal{H}_{1}\left(4 \mathcal{G}_{1}-\mathcal{G}_{2}\right)+\mathcal{H}_{2}\left(\mathcal{G}_{2}-\mathcal{G}_{1}\right)\right]$.

Using Eq. (37), one then finds

$\mathcal{A}^{\prime}(0)=\frac{2}{3} \frac{f_{V}^{2}}{M^{2}}$.

This matches the value obtained directly from Eq. (8),

$\mathcal{A}^{\prime}(0)=\int_{0}^{\infty} \mathrm{d} s \frac{\rho(s)}{s^{2}}=\frac{2}{3} \frac{f_{V}^{2}}{M^{2}}$. 
As a test of the expression for $a_{\mu}^{\text {hvp }}$, using Eq. (109) in the review [5], we obtain

$a_{\mu}^{\mathrm{hvp}}=\frac{8}{9} \alpha^{2} f_{V}^{2} \frac{m_{\mu}^{2}}{M^{2}} \hat{K}\left(M^{2}\right)$,

where the kernel $\hat{K}(s)$ appropriate for the timelike region is given in terms of elementary functions in [5]. Setting $M / m_{\mu}=2 m_{\pi^{ \pm}}=2.64187$, we have $\hat{K}\left(M^{2}\right)=0.63344$ and we thus obtain $a_{\mu}^{\text {hvp }}=0.0806733 \alpha^{2} f_{V}^{2}$. On the other hand, performing numerically the integral (69) with the $\mathcal{H}_{i}$ given by Eqs. (61-62) and the $\mathcal{G}_{i}$ read off from Eq. (67), we obtain the same value of $a_{\mu}^{\text {hvp }}$ to all indicated digits.

\subsection{Comparison with the time-momentum representation}

In order to prepare the discussion in the next section, it is interesting to compare the derived formulae with the timemomentum representation, in which only the spatial rotations are kept as manifest symmetries. The starting point in the TMR is the mixed-representation Euclidean correlator,

$G\left(x_{0}\right) \delta_{k \ell}=-\int \mathrm{d}^{3} x G_{k l}(x)$,

which has the spectral representation [19]

$G\left(x_{0}\right)=\int_{0}^{\infty} \mathrm{d} \omega \omega^{2} \rho\left(\omega^{2}\right) \mathrm{e}^{-\omega\left|x_{0}\right|}, \quad x_{0} \neq 0$.

The vacuum polarization and the Adler function can be expressed as an integral over $G\left(x_{0}\right)[19,21]$,

$$
\begin{aligned}
\Pi\left(Q_{0}^{2}\right)-\Pi(0) & \\
= & \int_{0}^{\infty} \mathrm{d} x_{0} G\left(x_{0}\right)\left[x_{0}^{2}-\frac{4}{Q_{0}^{2}} \sin ^{2}\left(\frac{1}{2} Q_{0} x_{0}\right)\right], \\
\mathcal{A}\left(Q_{0}^{2}\right) \equiv & Q_{0}^{2} \frac{\mathrm{d} \Pi}{\mathrm{d} Q_{0}^{2}}=\frac{1}{Q_{0}^{2}} \int_{0}^{\infty} \mathrm{d} x_{0} G\left(x_{0}\right) \\
& \left(2-2 \cos \left(Q_{0} x_{0}\right)-Q_{0} x_{0} \sin \left(Q_{0} x_{0}\right)\right) .
\end{aligned}
$$

In particular, the slope of the Adler function is given by

$$
\mathcal{A}^{\prime}(0)=\lim _{Q^{2} \rightarrow 0} \frac{\mathcal{A}\left(Q^{2}\right)}{Q^{2}}=\frac{1}{12} \int_{0}^{\infty} \mathrm{d} x_{0} x_{0}^{4} G\left(x_{0}\right) .
$$

The integrand for $\mathcal{A}^{\prime}(0)$ is displayed as a dashed curve in Fig. 2. Finally, the quantity $a_{\mu}^{\text {hvp }}$ is given by $[18,19]$

$$
\begin{aligned}
a_{\mu}^{\mathrm{HLO}}= & \left(\frac{\alpha}{\pi}\right)^{2} \int_{0}^{\infty} \mathrm{d} x_{0} G\left(x_{0}\right) \tilde{f}\left(x_{0}\right), \\
\tilde{f}\left(x_{0}\right)= & \frac{2 \pi^{2}}{m_{\mu}^{2}}\left(-2+8 \gamma_{\mathrm{E}}+\frac{4}{\hat{x}_{0}^{2}}-2 \pi \hat{x}_{0}\right. \\
& \left.+\hat{x}_{0}^{2}-\frac{8}{\hat{x}_{0}} K_{1}\left(2 \hat{x}_{0}\right)+8 \log \left(\hat{x}_{0}\right)+8 I_{p}\left(\hat{x}_{0}\right)\right),
\end{aligned}
$$

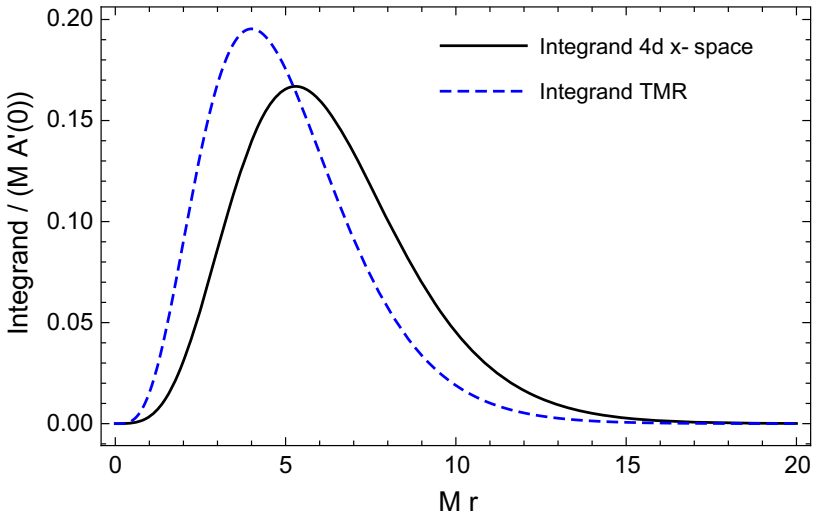

Fig. 2 The integrand in the integral over $|x|$ in Eq. (37) to obtain the Adler function at the origin, $\mathcal{A}^{\prime}(0)$, is displayed as a continuous curve. For comparison, the dashed curved is the integrand in the integration over Euclidean time in the time-momentum representation; see Eq. (77) with $\left(r \doteq x_{0}\right)$. In both cases, the model for the spectral function is given by Eq. (66) and the displayed functions are normalized such that the area under the curves is unity. Note that for $M=800 \mathrm{MeV}, M r=4$ corresponds to $1 \mathrm{fm}$

where $\hat{x}_{0}=m_{\mu} x_{0}$. Here $\gamma_{\mathrm{E}}=0.577216$.. is Euler's constant and

$I_{p}(z)=\frac{\pi z}{4}+\frac{1}{8} G_{1,3}^{2,1}\left(\left.z^{2}\right|_{0,1, \frac{1}{2}} ^{\frac{3}{2}}\right)$.

One aspect that is common between the CCS method and the TMR method is that, in continuum $\mathrm{QCD}$, the integrand for $\mathcal{A}^{\prime}\left(Q^{2}\right), \Pi\left(Q^{2}\right)-\Pi(0)$ and $a_{\mu}^{\text {hvp }}$ is of order $|x|$ (respectively order $\left.x_{0}\right)$ at short distances.

\section{Lattice QCD aspects}

In this section we discuss some of the possible implementation strategies of the covariant coordinate-space method. In the master relation (19), the $O(4)$ symmetry of the integrand in the continuum allows for a high degree of flexibility when implementing an estimator for $a_{\mu}^{\text {hvp }}$ in lattice QCD. The finite lattice spacing as well as the toric boundary conditions break the $O(4)$ symmetry. Therefore, for a number of classes $\Omega$ of lattice vectors on a lattice of dimensions $L^{4}$, it is useful to investigate consistency and statistical precision of the estimators

$a_{\mu}^{\text {est }}(\Omega)=\left.2 \pi^{2} \frac{\left|\varepsilon^{(\cdot)}\right|}{|\Omega|} \sum_{k=1}^{|\Omega|} \sum_{n=1}^{n_{\max }}\left[|x|^{3} G_{\mu \nu}(x) H_{\mu \nu}(x)\right]\right|_{x=n \varepsilon^{(k)}}$

where the lattice vectors $\varepsilon^{(k)}$ belong to an orbit $\Omega$ of the hypercubic group (for instance, $\Omega=\{a( \pm 1, \pm 1, \pm 1,0)$, $a( \pm 1, \pm 1,0, \pm 1), \ldots\}$, for which $|\Omega|=32$ and $\left|\epsilon^{(.)}\right|=$ $a \sqrt{3}$ ). Of course, a more sophisticated integration scheme 
may also be applied. If a $T \times L^{3}$ lattice is used $(T \neq L)$, the orbit is restricted to vectors related by the three-dimensional hypercubic symmetry group.

One may wonder how the signal-to-noise will compare between a position-space and the momentum-space method in a lattice QCD simulation. We argue that, in a large volume, one may expect an advantage with the covariant coordinatespace method. The reason is simple and stems from the fact that the 'signal', $G_{\mu \nu}(x) \sim \mathrm{e}^{-M|x|}$, falls off faster than the square-root of its variance $\sigma$. The latter is expected to drop only like $\sigma \sim \mathrm{e}^{-m_{\pi}|x|}$ for the isovector contribution. In the case of disconnected diagrams, $\sigma$ is asymptotically independent of $|x|$ with the standard algorithm. With the proposed CCS method, one may sum in the variable $|x|$ up to a maximum $R$ (effectively performing a weighted average over all orbits),

$a_{\mu}^{\mathrm{eff}}(R)=a^{4} \sum_{x:|x|<R} G_{\mu \nu}(x) H_{\mu \nu}(x)$,

with $|x|$ the Euclidean norm of the position vector $x$. The truncation distance $R$ is chosen so that the incurred error is sufficiently small. In practice, an extrapolation to $R=\infty$ based on Eq. (67), or a more sophisticated version involving the two-pion continuum, may be used. The important point is that only those points in $x$ are included in the sum which contribute up to a certain precision. By contrast, in a momentum-space method, the input data $\Pi_{\mu \nu}(Q)$ already involves a sum over the correlator $G_{\mu v}(x)$ over the whole volume, even though points very distant from the origin end up barely contributing to $a_{\mu}^{\text {hvp }}$. We therefor expect the CCS method to be superior.

In the TMR method, the variable $x_{0}$ plays a role analogous to $|x|$ in the CCS method. The sum in $x_{0}$ is truncated at some $x_{0}^{\max }$, beyond which the correlator is estimated using an extrapolation based on its spectral representation. Although less severe than in the four-momentum space method, the unfavorable aspect that spatially very distant points $\left(\left[\sum_{i=1}^{3} x_{i}^{2}\right]^{1 / 2}\right.$ large) are included in the estimator for $a_{\mu}^{\text {hvp }}$ remains present. The CCS method should be at an advantage here, particularly for disconnected diagram contributions computed on a large volume.

Having made this point, there are other considerations that contribute to choosing a computational method. One important consideration is the control over finite-volume effects. The nature of these corrections are by now quite well understood in the TMR method [18], although more direct numerical studies (involving several volumes, all other parameters being held fixed) are desirable. It remains to be studied how large the finite-size effects are in the CCS method. If the spatial torus size is $L$, the sum (82) would have to be truncated at $R_{\max } \leq L / 2$. However, one may be able to probe the long-distance part of the correlator further, since one can choose for instance the orbit of the vector $\varepsilon=a \cdot(1,1,1,1)$ and apply the estimator ( 81 ) for distances beyond $R_{\max }$. This procedure effectively extends the distance reach by a factor of two, at the cost of having less volume averaging.

Finally, we remark that the framework presented could also be used to calculate the transverse part of the axialcurrent correlator. In particular, the explicit projection onto the transverse part via the tensor $H_{\mu \nu}(x)$ takes care of removing the pion pole present in the longitudinal channel.

Acknowledgements This work was supported in part by DFG through CRC 1044 The low-energy frontier of the Standard Model.

Open Access This article is distributed under the terms of the Creative Commons Attribution 4.0 International License (http://creativecomm ons.org/licenses/by/4.0/), which permits unrestricted use, distribution, and reproduction in any medium, provided you give appropriate credit to the original author(s) and the source, provide a link to the Creative Commons license, and indicate if changes were made. Funded by SCOAP ${ }^{3}$.

\section{References}

1. F. Jegerlehner, Z. Phys. C 32, 195 (1986)

2. M. Eads, PoS FPCP2015, 046 (2015). arXiv: 1512.07214

3. E-989 Collaboration, http://muon-g-2.fnal.gov/

4. JPARC Experiment E34, http://g-2.kek.jp/

5. F. Jegerlehner, A. Nyffeler, Phys. Rep. 477, 1 (2009). arXiv:0902.3360

6. T. Blum, A. Denig, I. Logashenko, E. de Rafael, B. Lee Roberts, et al. (2013). arXiv: 1311.2198

7. T. Blum, Phys. Rev. Lett. 91, 052001 (2003). arXiv:hep-lat/0212018

8. M. Göckeler et al., QCDSF Collaboration. Nucl. Phys. B 688, 135 (2004). arXiv:hep-lat/0312032

9. C. Aubin, T. Blum, Phys. Rev. D 75, 114502 (2007). arXiv:hep-lat/0608011

10. X. Feng, K. Jansen, M. Petschlies, D.B. Renner, Phys. Rev. Lett. 107, 081802 (2011). arXiv: 1103.4818

11. P. Boyle, L. Del Debbio, E. Kerrane, J. Zanotti, Phys. Rev. D 85, 074504 (2012). arXiv: 1107.1497

12. M. Della Morte, B. Jäger, A. Jüttner, H. Wittig, JHEP 1203, 055 (2012). arXiv:1112.2894

13. F. Burger, X. Feng, G. Hotzel, K. Jansen, M. Petschlies, D.B. Renner (ETM), JHEP 02, 099 (2014). arXiv: 1308.4327

14. T. Blum, P.A. Boyle, T. Izubuchi, L. Jin, A. Jüttner, C. Lehner, K. Maltman, M. Marinkovic, A. Portelli, M. Spraggs, Phys. Rev. Lett. 116, 232002 (2016). arXiv: 1512.09054

15. T. Blum, et al. (RBC/UKQCD), JHEP 04, 063 (2016) (erratum JHEP 05, 034, 2017). arXiv: 1602.01767

16. B. Chakraborty, C.T.H. Davies, P.G. de Oliviera, J. Koponen, G.P. Lepage (2016). arXiv:1601.03071

17. S. Borsanyi, Z. Fodor, T. Kawanai, S. Krieg, L. Lellouch, R. Malak, K. Miura, K.K. Szabo, C. Torrero, B. Toth (2016). arXiv: 1612.02364

18. M. Della Morte, A. Francis, V. Gülpers, G. Herdoiza, G. von Hippel, H. Horch, B. Jäger, H.B. Meyer, A. Nyffeler, H. Wittig (2017). arXiv: 1705.01775

19. D. Bernecker, H.B. Meyer, Eur. Phys. J. A 47, 148 (2011). arXiv: 1107.4388 
20. X. Feng, S. Hashimoto, G. Hotzel, K. Jansen, M. Petschlies, et al. (2013). arXiv: 1305.5878

21. A. Francis, B. Jäger, H.B. Meyer, H. Wittig, Phys. Rev. D 88, 054502 (2013). arXiv: 1306.2532

22. A. Francis, V. Gülpers, B. Jäger, H. Meyer, G. von Hippel, et al., PoS LATTICE2014, 128 (2014). arXiv: 1411.7592

23. V. Gülpers, H. Meyer, G. von Hippel, H. Wittig, PoS LATTICE2015, 263 (2016)
24. T. Kawanai, S. Borsanyi, Z. Fodor, S. Krieg, L. Lellouch, R. Malak, K. Miura, K. K. Szabo, C. Torrero, B.C. Thot (Budapest-MarseilleWuppertal), PoS LATTICE2016, 171 (2017)

25. M. Knecht, Lect. Notes Phys. 629, 37 (2004). arXiv:hep-ph/0307239 